\title{
Musculoskeletal and Sports Medicine Physical Medicine and Rehabilitation Curriculum Guidelines
}

\author{
Jason L. Zaremski, MD, Matthew C. Diamond, MD, PhD, Andrea Aagesen, MD, \\ Ellen Casey, MD, Brian Davis, MD, Mark Ellen, MD, Kenneth Mautner, MD, \\ Kelly McInnis, MD, Jerome Nichols, MD, Ashwin Rao, MD, Brian Krabak, MD, MBA
}

\section{Introduction}

The following Musculoskeletal and Sports Medicine Physical Medicine and Rehabilitation (PM\&R) Curriculum Guidelines were developed to define a recommended training strategy for PM\&R residents in the realm of musculoskeletal and sports medicine. This document has been endorsed by the American Medical Society for Sports Medicine, the American Academy of Physical Medicine and Rehabilitation, and the Association of Academic Physiatrists. There are other published educational resources for sports medicine faculty that provide direction in improving the quality of sports medicine fellowship programs [1]. However, this document was developed to meet the unique needs and skills of the physiatrist. As is the case for all areas of physiatric training, the knowledge and skills in the areas of musculoskeletal and sports medicine should be attained through longitudinal experience that promotes educational competencies defined by the Accreditation Council for Graduate Medical Education (http://www.acgme.org) [2]. The curriculum guidelines recommend structured experiences in several areas unique to ambulatory physiatry training as well as structured didactic lectures, conferences, journal clubs, and workshops with an emphasis on outcomes-oriented, evidence-based studies.

\section{Overview}

\section{Goals of the Task Force}

The curriculum guidelines were developed through consensus recommendations of members of the Physiatry Curriculum Subcommittee of the American Medical Society for Sports Medicine Education Committee. The guidelines aim to assist residency programs in defining their own learning objectives and clinical experiences relevant to discipline-specific sports medicine education, with the understanding that many residencies provide limited sports medicine resourced to their trainees over the course of their residency. Such experiences include on-field medical care, team physician experience, training room exposure, musculoskeletal ultrasound, and direct work with sports medicine specialists in the ambulatory setting. Each residency program is responsible for its own curriculum and should consider using these guidelines as a means of informing its own sports medicine curriculum.

\section{Scope and Organization of the Guidelines}

This document is organized into 4 major topic areas with a physiatric focus: (1) musculoskeletal medicine; (2) other physiatric areas of sports medicine; (3) basic science foundations of sports medicine; and (4) special topics within sports medicine. The musculoskeletal medicine section is organized by body region. For each body region, learning objectives should include the performance of an appropriate history and physical examination (H\&P) and demonstration of familiarity with relevant diagnostic imaging and treatment modalities. A detailed description of these goals is beyond the scope of this document. Rather, for each body region, these guidelines provide specific, highyield learning objectives related to the most important musculoskeletal conditions and physiatric interventions for that region. Electrodiagnostic medicine and musculoskeletal ultrasound are addressed in individual sections. Resources published elsewhere for the delineation of specific objectives for H\&P and diagnostic imaging could include various PM\&R-focused textbooks. 


\section{Outline}

\section{Musculoskeletal Medicine}
A. Cervical Spine Region
B. Shoulder Region
C. Elbow/Forearm Region
D. Wrist/Hand Region
E. Thoracic Spine/Chest Region
F. Lumbar Spine Region
G. Pelvis/Hip Region
H. Knee Region
I. Ankle/Foot Region

\section{Other Physiatric Areas of Sports Medicine}

A. Concussion and Brain Injury

B. Spinal Cord Injury

C. Sports Cardiology and Exercise Physiology

D. Nutrition, Metabolism, and Endocrine Issues

E. Women's Health, Including Female Athlete Triad

F. Pediatric Sports Medicine

G. The Aging Athlete

$\mathrm{H}$. Electrodiagnostics

I. Prosthetics and Orthotics in Sports

J. The Disabled Athlete

K. Exercise as Medicine and Integrative Sports Medicine

L. Eating Disorders in Athletes

\section{Basic Science Foundations of Sports Medicine}
A. Pathophysiology of Tissues and Organs
B. Fundamental Biomechanical Principles
C. Imaging Fundamentals
D. Laboratory Studies
E. Commonly Used Medications

\section{Special Topics Within Sports Medicine}
A. Pre-participation Physical Examinations (PPE)
B. Sideline Coverage and Emergencies
C. Primary Care Sports Medicine Issues
D. Sports Dermatology
E. Musculoskeletal Ultrasound
F. Communication and Teamwork
G. Ethical and Medico-Legal Aspects and Profession- alism Serving as Team Physician
H. Mental Health and the Athlete

\section{Guidelines}

\section{Musculoskeletal Medicine}

\section{A. Cervical Spine Region}

1. H\&P and imaging. Perform an appropriate H\&P and demonstrate familiarity with cervical anatomy and incorporating the fundamentals of diagnostic imaging (radiograph, computed tomography [CT], and magnetic resonance imaging $[\mathrm{MRI}])$ of the cervical spine.

2. Cervical conditions. For each condition, describe typical clinical presentation, diagnostic criteria, and nonsurgical and surgical management, including rehabilitation prescription, as appropriate. Additional objectives are listed to follow.

a. Cervical strain and sprain. Identify the origins, insertions, actions, and innervations of the cervical muscles. Identify the ligaments of the cervical spine and how each contributes to the static stability. Describe the role of imaging with flexion-extension radiographs. Describe the role of cervical orthoses in the acute management of cervical strains. Describe a rehabilitation program, including postural re-education, functional restoration, and neck stretching and strengthening to exercises for athletes who sustain a neck strain or sprain without any instability.

b. Ligamentous injury/instability. Describe the radiologic criteria for instability on flexion/extension radiographs. Describe the specific consideration for patients with Down Syndrome, including the role of screening radiographs and sports-specific restriction. Describe Klippel-Feil syndrome and the role of imaging and considerations for sports participation for these patients.

c. Cervical spinal stenosis. Describe the clinical presentation of patients with spinal stenosis. Understand how to calculate and interpret the Torg/Pavlov ratio, know the uses and limitations of this measurement with respect to clearance for contact sports participation.

d. Cervical fractures. Describe the mechanism and management of each of these specific fractures to the cervical spine: Jefferson fracture, posterior arch fracture of C1, Hangman's fracture, odontoid fractures (types I-III), vertebral body compression fractures (type $\mathrm{I}-\mathrm{V})$. Please also describe the phases of fracture healing.

e. Transient quadriparesis/cervical cord neurapraxia. Describe the typical clinical presentation, mechanism, incidence, and recurrence rate of transient quadriparesis. Describe the on-field management of sports-related quadriparesis and neurapraxia. Describe the considerations related to return to play for patients with a single episode compared with repeated episodes of transient quadriparesis.

f. Cervical radiculitis/radiculopathy. Thorough understanding of the neuroanatomy of the cervical spine, spinal nerve roots, and brachial plexus; describe the typical presentation of cervical radiculitis and/or radiculopathy based on root level involvement. Describe the expected electrodiagnostic findings of a cervical radiculopathy at 1 week, 3-4 weeks, 5-6 weeks, and greater than 6 months. Contrast the electrodiagnostic findings of cervical radiculopathy with those observed with a nerve root avulsion or burner/stinger. Describe the indications for a cervical epidural steroid injection and for surgical interventions. 
g. Cervical neurapraxia (burners/stingers/cervical radicular impingement). Describe the typical mechanism of transient neurapraxia of the brachial plexus (burners/stingers/cervical radicular impingement). Describe the estimated incidence of this injury among football players and which nerve roots most often are affected. Describe the role for, including the appropriate timing of, electrodiagnostic studies for evaluation and prognosis for a burner/stinger. Describe the typical treatment and the considerations for role of return to play.

h. Cervical zygapophyseal (facet) arthropathy. Identify the most commonly affected joints of the cervical spine and describe their typical pain referral pattern.

i. Cervical spinal cord injury. See separate section entitled "Spinal Cord Injury."

3. Interventions. For each physiatric intervention, describe the approach, imaging modality (as appropriate), indications, and precautions.

a. Cervical zygapophyseal (facet) joint injections.

b. Cervical epidural injections.

c. Cervical region trigger point and other intramuscular injections.

\section{B. Shoulder Region}

1. H\&P and imaging. Perform an appropriate H\&P and demonstrate familiarity with the fundamentals of diagnostic imaging (radiography, CT, ultrasound, and MRI) of the shoulder.

2. Shoulder conditions. For each condition, describe typical clinical presentation, diagnostic criteria, and nonsurgical and surgical management, including rehabilitation prescription, as appropriate. Additional objectives follow.

a. Scapular dyskinesis and scapular winging. Identify the scapular stabilization muscles and describe their role in optimizing scapulothoracic function. Discuss the relevance of evaluating kinetic chain mechanics and sport specific technique in an athlete with shoulder pain. Describe the presentations and causes of lateral versus medial scapular winging.

b. Acromioclavicular (AC) joint sprains. Describe the structure of the $A C$ joint and the classification of $A C$ injury. Review which classes of $A C$ joint sprains require a surgical consultation.

c. Shoulder instability and dislocation. Describe the typical causes and presentations of anterior versus posterior shoulder dislocations. Discuss potential associated conditions, including Bankart and Hill-Sachs lesions and neurovascular injury. Describe a clinical approach to the hypermobile athlete. Understand the risk of recurrence for first-time shoulder dislocations in the young athlete and describe age-based risks of redislocation in a patient with an initial dislocation.

d. Rotator cuff (RTC) tendinopathy, RTC tears, and shoulder impingement syndrome. Identify the RTC muscles and discuss their function. Describe the mechanism of and contributing factors to impingement. Describe the natural history of RTC tears and indications/contraindications for surgical repair. Discuss the importance of evaluating the neck in patients with shoulder problems.

e. Bicipital tendinopathy and proximal biceps tendon rupture. Identify the anatomic course of the biceps tendon, including its origins and insertions. Review the pathophysiology and clinical manifestations of biceps tendinosis and rupture, as well as typical management strategies.

f. Labral pathology, including SLAP (ie, Superior Labral tear Anterior to Posterior) lesion. Describe the structure and function of the glenoid labrum.

g. Adhesive capsulitis. Describe what distinguishes adhesive capsulitis from other shoulder problems, its predisposing factors, typical clinical examination findings, and its natural history.

h. Parsonage-Turner syndrome and other brachial plexopathies. Delineate the brachial plexus and the clinical features of these syndromes. Discuss the electrodiagnostic evaluation of these conditions as well as management strategies.

i. Suprascapular neuropathy. Describe the common clinical presentation for suprascapular neuropathy, which muscle(s) are typically weakened, and how diagnosis is made using electromyography.

j. Thoracic outlet syndrome. Describe the various mechanisms of injury in supraclavicular, subclavicular, costoclavicular, and infraclavicular region. Identify the various presentations for each type of thoracic outlet syndrome, namely neural, arterial, and venous compression symptoms, and understand that compression can be postural/functional.

k. Glenohumeral arthritis. Discuss the clinical presentation, risk factors, and diagnostic criteria for this condition, as well as typical management strategies depending on the severity of clinical disease

l. Fractures of the shoulder region.

i. Clavicular fracture. Explain where clavicle fractures most commonly occur and the amount of shortening and displacement acceptable to treat nonoperatively.

ii. Scapular fracture. Determine whether the fracture is extra-articular or intra-articular. Determine whether the fracture involves the acromion, coracoid, or glenoid. Be aware of the common medical conditions that can be associated with this injury, including injury to the pulmonary system.

iii. Proximal humerus fracture. Review the Neer classification scheme and consider management based on degree of displacement.

3. Interventions. For each intervention, describe the approach, imaging modality used (as appropriate), indications, and precautions.

a. Subacromial injection.

b. Bicipital tendon sheath injection. 
c. Glenohumeral joint injection.

d. AC joint injection.

e. Suprascapular nerve block.

f. Quadrilateral space/axillary nerve block.

g. Shoulder joint reduction.

\section{Elbow/Forearm Region}

1. H\&P and imaging. Perform an appropriate H\&P and demonstrate familiarity with the fundamentals of diagnostic imaging (radiograph, CT, ultrasound, and MRI) of the elbow.

2. Elbow conditions. For each condition describe typical clinical presentation, diagnostic criteria, and nonsurgical and surgical management, including rehabilitation prescription, as appropriate. Additional objectives follow.

a. Lateral epicondylopathy. Identify the most commonly involved muscle. Review the different treatment algorithms for acute epicondylitis versus chronic lateral epicondylosis. Review the most recent literature on corticosteroid injections and orthobiologics (such as platelet-rich plasma, stem cells, bone marrow aspirate concentrate) and percutaneous needle fenestration, and their short-term versus long-term efficacy.

b. Medial epicondylopathy. Identify the most commonly involved muscle. Review the differences in management of acute versus chronic medial epicondylitis.

c. Olecranon bursitis. Discuss the differences between acute and chronic olecranon bursitis.

d. Distal biceps tendon rupture. Describe the most common mechanism of this injury. Articulate why surgical repair is recommended for this injury when it is not generally recommended for proximal bicep tendon disruption.

e. Triceps tendinitis. Discuss the most common causes and what preventive measures should be undertaken to decrease risk of recurrence.

f. Ulnar collateral ligament (UCL) injury of the elbow. Describe the biomechanical importance of the $\mathrm{UCL}$ in relation to overhead throwers. Review the gold standard imaging modality to diagnose this injury. Explain what preventative measures are used to decrease risk of this injury.

g. Valgus extension overload syndrome/posterolateral rotatory instability. Know the overhand pitching cycle and review the phases and events of throwing. Describe the biomechanical importance of the UCL in relation to overhead throwers.

h. Medial epicondylar apophysitis (little league elbow). Discuss the importance of rest and pitch restrictions and how this impacts adolescent elbow throwing injuries. State the order of epiphyseal closure of the growth plates in the adolescent elbow, with ages.

i. Elbow dislocations. Review the classification scheme of elbow dislocations. Differentiate between simple versus complex dislocations. Understand the importance of a timely neurovascular examination. j. Radial head subluxation (nursemaid's elbow). Describe the mechanism of injury and why the size of the radial head diameter plays a role.

k. Osteochondritis dissecans (OCD) of the elbow. Describe the typical location of OCD of the elbow. Differentiate the treatment algorithm in displaced versus nondisplaced lesions. Describe the difference between OCD of the elbow and Panner disease (see the section "Pediatric Sports Medicine").

l. Volar compartment syndrome (Volkmann ischemia). Review the fractures that are associated with this syndrome. Discuss the most common of the 6 "Ps" associated with this injury. Explain why this type of injury is a potential surgical emergency.

m. Nerve injury

i. Anterior interosseous nerve (AIN) Syndrome. Review the AIN innervated muscles. Predict the common signs and symptoms of AIN syndrome. Prepare an electromyographic study to evaluate for AIN injury.

ii. Pronator syndrome. Discuss the potential sites of compression of the median nerve. Prepare an electromyographic study to evaluate for this syndrome.

iii. Posterior interosseous nerve (PIN) syndrome. Review the PIN innervated muscles. Predict the common signs and symptoms of a patient that may have PIN syndrome. Explain why there is no sensory component associated with this syndrome. Please explain why radial tunnel syndrome may present with sensory symptoms whereas PIN syndrome may present with sensory and motor symptoms.

iv. Cubital tunnel syndrome. Describe the type of elbow fractures associated with this type of nerve injury.

n. Fractures of the elbow region.

i. Distal humerus.

ii. Radius.

iii. Ulna.

3. Interventions. For each physiatric intervention, describe the approach, imaging modality (as appropriate), indications, and precautions.

a. Elbow joint injection and/or aspiration.

b. Olecranon bursa aspiration and/or injection.

c. Lateral epicondylopathy injection.

d. Medial epicondylopathy injection.

e. Radial head subluxation.

f. Posterior elbow dislocation reduction.

g. Cubital tunnel hydrodissection.

h. Radial tunnel injection/hydrodissection.

i. Elbow dislocation relocation techniques.

j. Radial head subluxation/dislocation reduction techniques.

\section{Wrist/Hand Region}

1. H\&P and imaging. Perform an appropriate H\&P and demonstrate familiarity with the fundamentals of diagnostic imaging (radiograph, CT, ultrasound, and MRI) of the wrist/hand. 
2. Wrist and hand conditions. For each condition describe typical clinical presentation, diagnostic criteria, and nonsurgical and surgical management, including rehabilitation prescription, as appropriate. Additional objectives are below.

a. Tendon/cartilage injury.

i. De Quervain tenosynovitis. Identify the involved tendons. Review the contents of each dorsal wrist compartment.

ii. Intersection syndrome. Identify the involved tendons. Explain the difference, clinically, between this syndrome and De Quervain tenosynovitis.

iii. Mallet finger and jersey finger. Describe the mechanisms of injury. Describe what is seen on clinical examination in each case. Explain the treatment algorithm and justify nonsurgical management versus surgical management for each.

iv. Trigger finger. Describe the natural history of this syndrome and the cause of the mechanical catching. Discuss treatment options, including collagenase injections.

v. Boutonniere deformity. Describe the mechanism of injury, what structure is injured, and the treatment algorithm in acute and chronic (ie, $>6$ weeks) cases.

vi. Triangular fibrocartilage complex injury. Describe the clinical importance of positive versus negative ulnar variance and how it relates to the triangular fibrocartilage complex. Describe the many potential mechanisms of injury. Review the gold standard imaging modality. Explain when surgical consultation is indicated.

vii. Scapholunate ligament injury. Describe the common mechanism for this injury and associated fracture(s). Explain the utility of the Watson test when assessing for this injury on physical examination. Describe the common abnormal findings on radiographs with a clenched fist view and lateral radiographs. Understand the importance of a surgical referral in an acute non-degenerative injury.

\section{b. Ligamentous injury.}

i. First metacarpal UCL (skier's or gamekeeper's thumb). Review the mechanism of injury and explain why it is important to image the injury prior to stressing the thumb. Define a Stener lesion and how the presence of one can change treatment decisions. Describe treatment options and their time frames.

c. Fractures of the wrist/hand region.

i. Distal radius/ulnar. Describe the most common mechanism of injury. Explain when to use a short-arm cast versus a long-arm cast. Review the indications for surgical versus non-operative management.

ii. Scaphoid. Describe the appropriate radiologic views to evaluate for this injury and discuss the role of radiographs in diagnosing scaphoid fracture. Describe the anatomy of the scaphoid and its blood supply and how location of fracture could affect healing and management decisions. iii. Metacarpal. Describe how to look for shortening on examination. Review the amount of angulation required to perform a reduction per digit. State the amount of shortening in oblique and spiral fractures that require surgical fixation. Explain the differences between a Bennett fracture and Rolando fracture.

d. Common dislocations.

i. Distal radioulnar joint. Describe the most common mechanism of injury. Explain when nonoperative treatment is acceptable.

ii. Interphalangeal. Explain why dorsal dislocation is more common than volar for proximal interphalangeal dislocations. Why are athletes in contact sports most at risk? Describe the most common mechanism of injury for distal interphalangeal dislocations. Explain the association of a distal interphalangeal dislocation with a mallet finger.

e. Miscellaneous injury.

i. Ganglion cysts. Describe the most common location to find this cyst. Describe the treatment algorithms for conservative and operative management. Review the imaging modalities to confirm diagnosis.

ii. Subungual hematoma. Explain why a radiograph should be ordered with any acute hematoma of the nail bed. Describe the procedure of how to drain this type of a hematoma.

iii. Hook of hamate fracture. Racket sports, very relevant to sports

iv. OCD of the lunate/Kienbock's should be considered.

f. Nerve injury.

i. Carpal tunnel syndrome (CTS). Review the median nerve innervated hand muscles. Predict the common signs and symptoms of a patient that may have CTS. Describe all the special physical examination tests that are used to assess for CTS. Differentiate on physical examination between CTS and cervical radiculopathy. State what the most common risk factors and causes are to develop CTS.

ii. Ulnar nerve entrapment (UNE) at the wrist (handlebar palsy). Review the ulnar nerve innervated hand and forearm muscles. Predict the common signs and symptoms of a patient who may have UNE. Describe all the special physical examination tests assessing for UNE. Differentiate on physical examination between UNE at the wrist versus the elbow (cubital tunnel syndrome). State what the most common risk factors and causes are to develop UNE.

3. Interventions. For each physiatric intervention, describe the approach, imaging modality (as appropriate), indications, and precautions.

a. Carpal tunnel injection.

b. De Quervain tenosynovitis injection.

c. Carpometacarpal joint injection.

d. Trigger finger injections.

e. Ganglion cyst aspiration. 


\section{E. Thoracic Spine/Chest Region}

1. H\&P and imaging. Perform an appropriate H\&P and demonstrate familiarity with the fundamentals of diagnostic imaging (radiograph, CT, and MRI) of the thorax.

2. Thoracic spine/chest musculoskeletal conditions. For each condition describe typical clinical presentation, diagnostic criteria, and nonsurgical and surgical management, including rehabilitation prescription, as appropriate. Additional objectives are below.

a. Thoracic spinal disorders.

i. Thoracic degenerative disc disease, including herniated nucleus pulposis (HNP). Describe why HNPs occur less frequently in the thoracic spine than in the lumbar or cervical spine.

ii. Thoracic vertebral compression fracture. Articulate a biomechanical theory of spinal support as well as a categorization system of spinal fractures. Describe the risks and benefits of conservative management versus interventional treatment.

iii. Scheuermann disease/kyphosis. Describe the specific diagnostic criteria of Scheuermann disease. Predict the common findings on radiographs. Recommend when bracing versus surgical referral is recommended based on the extent of kyphotic angulation.

iv. Thoracic zygapophyseal arthropathy. Describe the typical pain patterns for these joints and the evidence for their role as pain generators.

v. Ankylosing spondylitis. Describe an appropriate workup for the spondyloarthropathies.

b. Costal disorders.

i. Rib fractures. Describe the differential presentation of rib stress fractures versus acute fracture, and complete versus incomplete injuries. Describe the mechanism of injury and presentation for flail chest. Know the treatment and prognosis for return to activity.

ii. Costovertebral and costotransverse disorders. Describe costochondritis, costochondral sprain, and separation.

\section{c. Sternal disorders.}

i. Sternoclavicular sprains, subluxations, and dislocations. Describe the differences between firstversus second-degree sprains and Type I, II, and III subluxations/dislocations, as well as the relative dangers of whether the latter is anterior versus posterior.

d. Soft-tissue disorders.

i. Pectoral strains and tears. Describe a typical mechanism of injury, the different anatomical sites of injury, and the role of conservative versus surgical treatment. Describe a presentation that would be more likely to be associated with performance-enhancing drugs.

e. Disorders of the vasculature and vital organs.

i. Aortic aneurysm.

ii. Splenic rupture.

3. Interventions. For each physiatric intervention, describe the approach, imaging modality (as appropriate), indications, and precautions. a. Thoracic zygapophyseal (facet) joint injections.

b. Costovertebral injections

c. Thoracic epidural injections.

d. Thoracic region trigger point and other intramuscular injections.

\section{F. Lumbar Spine Region}

1. H\&P and imaging. Perform an appropriate H\&P and demonstrate familiarity with the fundamentals of diagnostic imaging (radiographs, CT, and MRI) of the lumbar spine.

2. Lumbar spine conditions. For each condition describe typical clinical presentation, diagnostic criteria, and non-surgical and surgical management, including rehabilitation prescription, as appropriate. Additional objectives follow.

a. Spondylolysis. Identify the different types, the reliability of different imaging modalities in making this diagnosis, and management options and return-to-play guidelines.

b. Spondylolisthesis. Describe the different clinical causes, grading of this condition, the natural history, and management options.

c. Spondylosis. Describe the normal anatomy and physiology of the spine and the degenerative cascade (dysfunction, instability, and stabilization). Understand common radiographic finding and utility of advanced imaging and electrodiagnostic testing. Know the proper use of bracing, injections, and corestrengthening techniques, and understand return to play criteria.

d. Spinal stenosis. Describe central versus neuroforaminal spinal stenosis, including clinical presentation and indications for surgical management.

e. Annular fissure. Understand the anatomy of the annulus, including fiber alignment, blood supply, and innervation. Know the mechanism of injury and natural history.

f. HNP. Describe the myriad pain patterns that can be elicited from an HNP. Describe the prevalence of asymptomatic HNP in the general population as a function of age.

g. Lumbosacral radiculitis/radiculopathy. Describe the typical dermatomal pain patterns and myotomal weakness and/or reflex changes at the levels of the lumbosacral spine.

h. Mechanical causes of lumbar pain. Understand anatomy and presentation of mechanical low back pain and importance of "core strength" and lower extremity flexibility. Understand how errors in training, technique, and improper equipment can predispose to injury.

i. Medical causes of lumbar pain. Understand and describe medical illness' that can cause lumbar pain including rheumatologic renal, pancreatic, hepatic, vascular, pulmonary, hematologic including splenic, malignant and non-malignant etiologies, and infectious 
causes including discitis, osteomyelitis, sexually transmitted diseases, tuberculosis, etc.

j. Pediatric/youth injuries. Know the typical injuries associated with the skeletally immature, including spondylolysis, scoliosis; congenital anomalies not previously documented; tumors of childhood, Juvenile rheumatoid arthritis.

k. Lumbar vertebral fracture.

3. Interventions. For each physiatric intervention, describe the approach, imaging modality (as appropriate), indications, and precautions.

a. Lumbar zygapophyseal (facet) joint injection.

b. Lumbar medial branch block and radiofrequency ablation.

c. Lumbar epidural injections, including transforaminal, interlaminar, and caudal approaches.

d. Lumbar region trigger point and other intramuscular injections.

e. Sacroiliac joint injection.

\section{G. Pelvis/Hip Region}

1. H\&P and imaging. Perform an appropriate H\&P and demonstrate familiarity with the fundamentals of diagnostic imaging (radiograph, CT, ultrasound, and MRI) of the pelvis/hip.

2. Pelvis/hip musculoskeletal conditions. For each condition describe typical clinical presentation, diagnostic criteria, and nonsurgical and surgical management, including rehabilitation prescription, as appropriate. Additional objectives are below.

a. Femoroacetabular impingement (FAl). Describe the 2 types of FAl (cam, pincer) and how these deformities lead to abnormal contact between the femur and the acetabulum. Understand that the distribution of pain can be groin, lateral hip ("C sign") and/or in the posterior pelvis. Discuss the association of FAl with labral pathology. Understand the role of the diagnostic hip injection.

b. Hip labral tear. Recognize the prevalence of asymptomatic labral tears. Understand the relationship between labral tears and the development of early osteoarthritis. Discuss appropriate imaging modalities for labral tears (MRI-arthrogram versus 3-Tesla MRI versus ultrasound).

c. Osteoarthritis. Discuss the risk factors for hip osteoarthritis. Describe the role of aerobic exercises and strength training in management of hip osteoarthritis.

d. Sacroiliitis. Review common imaging findings on radiographs and MRI. Explain which laboratory studies should be ordered and expected results if positive. Explain treatment based on overuse versus infectionbased sacroiliitis.

\section{Extra-articular hip disorders}

a. Femoral neck stress fracture. Discuss the risk factors for femoral neck stress fractures. Describe the 2 types: compression-side versus tension-side and the implications for management.

b. Greater trochanteric pain syndrome (GTPS). Describe the diagnostic criteria for GTPS. Understand the importance of ruling out referred pain from the lumbar spine and the hip joint in patients with this presentation. Discuss the role of hip abductor weakness in GTPS.

c. Snapping hip. Describe the structures involved in the three types of snapping hip: external, internal and intra-articular.

d. Piriformis syndrome. Describe the pathophysiology of piriformis syndrome. Discuss how to differentiate between piriformis syndrome versus L5-S1 radiculopathy.

e. Ischiofemoral impingement. Describe the pathophysiology of ischiofemoral impingement. Explain how this syndrome results in damage to the quadratus femoris muscle.

4. Pelvic Disorders

a. Hip pointer. Discuss the muscles that insert onto the iliac crest. Describe physical examination or radiographic findings to help rule out an avulsion.

b. Athletic pubalgia. Described the primary sites of pathology. Discuss the proposed mechanism of injury.

c. Osteitis pubis. Describe the proposed underlying mechanism and radiographic finding associated with osteitis pubis.

d. Adductor strain. Discuss the grading scale for muscle strains. Describe the most common mechanism of injury.

e. Stress fractures. Describe the risk factors associated with pubic ramus and sacral stress fractures. Discuss the utility of radiographs versus MRI versus bone scan to diagnose pelvic stress fractures.

f. Sacroiliac joint (SIJ) dysfunction/pain. Discuss the risk factors for SIJ pain. Describe the utility of combining 4-5 provocative maneuvers used during the physical examination to diagnose SIJ pain.

g. Pelvic floor dysfunction/pain. Define the muscles that comprise the pelvic floor. Understand the manifestations of pelvic floor dysfunction in athletes, including stress urinary incontinence and pain.

5. Interventions. For each physiatric intervention, describe the approach, imaging modality (as appropriate), indications, and precautions.
a. Intra-articular hip injection.
b. SIJ injection.
c. Greater trochanteric bursa injection.
d. Iliopsoas bursa injection.
e. Pubic symphysis injection.

\section{H. Knee Region}

1. H\&P and imaging. Perform an appropriate H\&P and demonstrate familiarity with the fundamentals of diagnostic imaging (radiograph, CT, ultrasound, and MRI) of the knee. 
2. Knee conditions. For each condition describe typical clinical presentation, diagnostic criteria, and non-surgical and surgical management, including rehabilitation prescription, as appropriate. Additional objectives are below.

a. Patellofemoral pain syndrome (PFPS). Identify the underlying factors contributing to patellar tracking/ maltracking.

b. Iliotibial band syndrome. Describe some of the muscular imbalances and dysfunctions that may contribute to this syndrome. Describe the anatomy of the iliotibial band.

c. Plica syndrome/medial plica syndrome. Identify the features that help distinguish medial plica syndrome from PFPS or injuries to the medial meniscus.

d. Patellar tendinopathy and patellar tendon rupture. Describe the role of ultrasound in aiding in diagnosis of patellar tendinopathy.

e. Quadriceps tendinopathy and quadriceps tendon rupture.

f. Patellar subluxation/dislocation. Describe the common factors underlying PFPS and patellar dislocation. Describe some of the pathoanatomic variants that may contribute to recurrent episodes and failure of even surgical management if not adequately addressed.

g. Prepatellar/infrapatellar/supra-patellar bursitis. Review the typical causes of each type of bursitis. Also identify typical associated conditions.

h. Pes anserine bursitis/tendinitis. Describe the location and anatomic structures comprising the pes anserine.

i. Baker cyst/popliteal cyst. Describe the association between popliteal cysts and intra-articular pathologies such as osteoarthritis. Describe the typical location and anatomy of a Baker cyst, including the surrounding musculature.

j. Osteoarthritis. Explain why weight-bearing films are preferred to nonweight-bearing radiographs when evaluating knee osteoarthritis. Define what a "sunrise" view is and why it is obtained. Explain the role of viscosupplementation in the conservative management of knee osteoarthritis.

k. Meniscus tears. Differentiate the typical features of traumatic versus degenerative meniscus tears. Describe at least three provocative physical exam maneuvers for testing of meniscal injury. Describe the "double posterior cruciate ligament" sign. Explain why bucket handle tears require surgical referral and with what urgency. Explain what areas of the meniscus receive greater vascularity and its application to the management of meniscal injuries.

l. Medial and lateral collateral ligament tears. Differentiate management of tear based on severity of injury.

m. Anterior cruciate ligament $(A C L)$ tear. Be familiar with the role of biomechanical prevention programs in preventing $A C L$ injuries. n. Posterior cruciate ligament tear. Describe the role of nonoperative management for posterior cruciate ligament tears.

o. Posterolateral corner (PLC) injury. Know the relevant anatomy of the PLC. Identify the three most important static stabilizers of the posterolateral knee and what movements they restrict. Identify the 2 other important ligamentous structures commonly injured along with the PLC.

p. Tibial plateau fracture. Describe the associated neurovascular risks associated with medial tibial plateau fractures.

q. OCD. Describe the most common location for an OCD lesion in the knee.

r. Sinding-Larsen-Johansson disease and OsgoodSchlatter disease. Describe the typical patient population in which these conditions appear.

s. Fractures. Describe the common fractures about the knee.

3. Nerve injury: Peroneal nerve injury.

4. Vascular phenomena.
a. Popliteal artery entrapment.
b. Iliac artery endofibrosis.

5. Interventions. For each physiatric intervention, describe the approach, imaging modality (as appropriate), indications, and precautions.
a. Intra-articular knee joint injection.
b. Knee joint effusion aspiration.
c. Prepatellar bursa aspiration and injection.
d. Baker cyst aspiration.
e. Patellar reduction.
f. Peroneal nerve hydrodissection.

\section{Ankle/Foot Region}

1. H\&P and imaging. Perform an appropriate H\&P and demonstrate familiarity with the fundamentals of diagnostic imaging (radiograph, CT, ultrasound, and MRI) of the ankle/foot.

2. Ankle/foot conditions. For each condition describe typical clinical presentation, diagnostic criteria, and nonsurgical and surgical management, including rehabilitation prescription, as appropriate. Additional objectives follow.

a. Ankle.

i. Lateral ankle sprain. Identify the lateral ankle stabilizing ligaments and classification of injury. Discuss potential associated conditions such as anterior process calcaneus fracture, peroneal tendinopathy, sinus tarsi syndrome, intraarticular pathology.

ii. High ankle sprain (syndesmotic injury). Discuss mechanism of injury. Describe radiographic findings in unstable sprain.

iii. Peroneal tendinopathy. Describe anatomy and function of peroneus longus, brevis, superficial peroneal retinaculum. Describe examination maneuver for snapping peroneal. 
iv. Sinus tarsi syndrome. Describe anatomic confines of sinus tarsi.

v. Anterior ankle impingement. Discuss bony versus soft tissue impingement and risk factors.

vi. Posterior tibial tendinopathy. Discuss the classification of posterior tibial dysfunction and acquired flat foot deformity. Discuss orthotic/bracing options.

vii. Achilles tendinopathy/Retrocalcaneal bursitis. Differentiate anatomy and pathophysiology of Achilles peritenonitis, midsubstance tendinosis, insertional tendinopathy, and retrocalcaneal bursitis/subcutaneous bursitis.

viii. Posterior ankle impingement/Os Trigonum syndrome/flexor hallucis longus tendinopathy.

ix. Talar osteochondral injury.

$x$. Tarsal tunnel syndrome. Describe anatomy of the tarsal tunnel. Describe the innervation to the foot and ankle region, including the saphenous nerve, superficial and deep peroneal nerves, sural nerve, medial and lateral plantar nerves.

xi. Osteoarthritis of the tibiotalar joint. Classify severity based on the Takakura classification. Elaborate on nonoperative treatment options, including the use of the single rocker sole shoe and why it can improve symptoms. Indicate when a surgical referral is recommended.

b. Foot.

i. Hindfoot.

- Plantar fasciopathy. Describe the Windlass mechanism, the basic biomechanics of gait, and the purpose of an orthotic device for the foot.

- Calcaneal stress fracture.

- Talocalcaneal coalition.

ii. Midfoot.

- Navicular stress fracture. Please explain the concern with a stress fracture to the navicular bone with respect to its arterial supply. State where the "N" spot is located.

- Os Navicularis. Please explain what Os navicularis syndrome is. Be able to discuss appropriate radiographic workup and subsequent appropriate treatment regimen.

- Lisfranc injury. Describe anatomy of ligament complex. Mechanism of injury. Most frequently misdiagnosed injury of foot. Appropriate radiographic workup and determining stability of injury. Appropriate triage to orthopedic surgery.

iii. Forefoot.

- Metatarsal injury. For the fifth metatarsal fracture, describe 3 zones of injury, an avulsion fracture, a Jones fracture, and a dancer's fracture, including classification of injury and triage to orthopedic surgery.

- Morton neuroma.

- Frieberg infraction.

- Turf toe/first metatarsophalangeal (MTP) plantar plate sprain. Describe anatomy of region
- Hallux rigidus.

- Crystal-induced arthropathy.

- Gout/pseudogout.

- Sesamoid injury. Discuss sesamoiditis, sesamoid stress injury, and bipartite sesamoid.

- Hammer toe/claw toe.

- Hallux valgus deformity/bunion.

- Metatarsalgia.

- MTP synovitis.

3. Interventions. For each physiatric intervention, describe the approach, imaging modality (as appropriate), indications, and precautions.

a. Fracture management of the foot. casting.

i. Know the fundamentals of acute splinting and orthopedic service.

ii. Understand appropriate criteria for triage to

b. Common injections (with and without imaging guidance).

i. Tibiotalar.

ii. Subtalar.

iii. Peroneal tendon sheath.

iv. Sinus tarsi.

v. Achilles tendon brisement procedure.

vi. Retrocalcaneal bursa.

vii. Plantar fascia.

viii. Flexor hallucis longus tendon sheath.

ix. Os trigonum/posterior ankle impingement.

$x$. Tarsal-metatarsal joint.

xi. First MTP joint.

xii. Morton neuroma.

c. Compartment Pressure Testing of lower limb.

\section{Other Physiatric Areas of Sports Medicine}

\section{A. Concussion and Brain Injury}

Be aware of the most up-to-date guidelines on concussion in sport management recommendations [3].

1. Define concussion. Describe the mechanism of injury and pathophysiology of mild traumatic brain injury (primary and secondary injury). Describe the signs and symptoms.

2. Identify risk factors for injury and their role for a protracted course, including age; gender; genetics; sport; position; medical and psychiatric history, and history and nature of the injury (number of injuries, severity, and duration of symptoms).

3. Describe an appropriate evaluation of a concussion, including:

a. Preinjury testing: baseline testing/preparticipation examination (PPE).

b. Sideline evaluation and management.

i. Rule out catastrophic head/neck injury.

ii. Role of sideline assessment tools.

iii. Rules regarding prohibition of same-day re-

turn to play if concussion is suspected. 
c. Comprehensive clinic evaluation, including emotional.

i. Symptom inventory: physical, cognitive, sleep,

ii. Neurologic examination.

iii. Balance assessment.

iv. Mental status evaluation.

d. Role of neuropsychological testing.

e. Role of neuroimaging.

4. Fundamentals of clinical management.

a. Relative physical and cognitive rest.

b. Appreciate the importance of early light nonexertional exercise and its role in reducing duration of symptoms [3-5].

c. Role of medication management.

d. Vestibular therapy.

e. Return to academics.

f. Return to athletics through a graded exertional program [3].

5. Describe postconcussion syndrome, including the role of a multidisciplinary approach, medications, and an exercise prescription.

6. Be familiar with these fundamental concepts.

a. Second-impact syndrome.

b. Disqualification from sport.

c. Chronic traumatic encephalopathy.

7. Outreach efforts at the local and national levels.

a. Prevention, education, and legislation regarding concussion in sports.

b. Be able to direct patients and caregivers to their respective high school state's athletic association and concussion legislation.

c. Inform caregivers and patients that most state's concussion legislation requires a licensed medical provider to clear a player to return to play.

8. Discuss prevention strategies for concussion in sports.

a. Rule changes at state and collegiate levels.

b. Tackling technique.

c. Equipment modification.

\section{B. Spinal Cord Injury}

1. Define spinal cord injury. Compare and contrast the mechanism of injury, neurologic deficits, and prognosis of the following acute spinal cord injuries:

a. Complete spinal cord injury.

b. Anterior cord syndrome.

c. Central cord syndrome.

d. Brown-Séquard syndrome.

e. Cervical cord neurapraxia (transient quadriparesis or transient neurapraxia). Include the incidence and recurrence rate of transient quadriparesis. Describe the considerations related to return to play for patients with a single episode compared with repeated episodes of transient quadriparesis.

2. Tackling technique in contact sports. Understand the importance in contact sports of keeping one's head up to avoid hyperflexion of the cervical spine when tackling and/or making contact with an opponent and its association with spinal cord injury.

3 . Describe the on-field examination and management of suspected cervical spine injuries, including:

a. Describe the physical examination of an athlete with a suspected cervical spine injury and list signs and symptoms concerning for a spinal cord injury that necessitate immobilization.

b. List the sideline equipment that may be necessary for management of an athlete with a spinal cord injury.

c. Be aware of the emergency action plan (EAP) that each athletic trainer has for each respective school where a sport is played. If an EAP is not available or if there is not an athletic trainer, meet with emergency medical services personnel before a game or discuss by phone procedures for transfer to an emergency department should an emergency occur.

d. List the step-by-step procedure for spine boarding an athlete on the field. Contrast how this procedure differs for athletes wearing full football pads and helmet versus an athlete wearing football helmet without pads, versus an athlete who is not wearing equipment. Review the procedure for removing facemasks.

e. Describe how to evaluate an ambulatory athlete on the sideline for a suspected cervical spine injury. In an athlete with transient neurologic symptoms, what criteria should be met before considering return to play.

4. Describe the role of imaging for cervical injuries.

a. Anteroposterior and lateral, open-mouth odontoid radiographs.

b. Flexion and extension radiographs. Describe how cervical spasms may limit the sensitivity this test. Describe the radiologic criteria for instability on flexion/ extension radiographs.

c. MRI.

d. CT.

e. SCIWORA (Spinal Cord Injury Without Radiographic Abnormality). Define and describe its significance.

5. Describe how to perform an ASIA (American Spinal Injury Association) examination and how it relates to motor recovery prognosis. Identify the upper extremity muscles innervated by each cervical nerve root level.

6. Describe the presentation and management of autonomic dysreflexia, including which spinal cord injury levels are susceptible to autonomic dysreflexia. Describe how boosting is used by athletes with spinal cord injuries in competitive sports and how this is monitored/detected during competition.

\section{Sports Cardiology and Exercise Physiology}

1. Be comfortable performing a basic cardiopulmonary H\&P, including an auscultatory cardiac examination. 
2. Be familiar with the fundamentals of reading an electrocardiogram, identify electrophysiologic changes of the athletic heart and abnormalities (see below), and be aware of the international criteria for electrocardiogram interpretation in athletes [6].

a. Hypertrophic cardiomyopathy.

b. Long-QT syndrome.

c. Wolff-Parkinson-White syndrome.

d. Arrhythmogenic right ventricular cardiomyopathy.

3. Familiarize yourself with the standard PPE. See separate PPE section of these guidelines.

4. Know the physiologic effects of exercise on the cardiopulmonary system.

a. Understand the relationship between cardiac output, heart rate, and stroke volume.

b. Be familiar with the Fick equation.

c. Understand the differences in the acute cardiovascular and pulmonary effects of and cardiovascular adaption to static versus dynamic exercise.

d. Know the definition of $\mathrm{VO}_{\max }$ (ie, maximum rate of oxygen consumption) and how it changes with training.

e. Understand what adaptation is primarily responsible for increased cardiac output as a result of training.

f. Understand what pulmonary adaptations occur in trained individuals, including changes in respiratory rate at rest versus submaximal and maximal exercise, tidal volume, minute ventilation, and respiratory muscle strength.

g. Be familiar with morphologic changes in the heart associated with static and dynamic exercise. Review the law of Laplace.

h. Demonstrate a familiarity with athletic heart syndrome. Know the typical normal value range for left ventricular cavity size and septal thickness.

i. Be aware of changes in the cardiovascular system that occur after periods of deconditioning.

j. Be aware of approximate metabolic equivalents of task of common activities, and be aware of appropriate metabolic equivalents of task recommendations when prescribing exercise to individuals of varying fitness levels.

k. Be familiar with the role of perceived exertion/ the Borg Rating of Perceived Exertion scale in exercise prescription.

5. Congenital heart disease (CHD) and exercise.

a. Recognize the importance of obtaining a value for pulmonary artery pressure in directing exercise prescriptions in individuals with CHD.

b. Have an understanding of basic CHD terminology (eg, cyanotic versus acyanotic, shunt, tetralogy of Fallot, transposition, etc).

c. Be familiar with the concept of static versus dynamic exercise, and that different CHD conditions may permit high amounts of one versus the other.

d. Be aware of how certain types of CHD can be at increased risk for cardiac events in collision sports (eg, Marfan syndrome). e. Know the historical and physical examination findings typically present in shunt lesions (eg, ventricular septal defects, atrial septal defects, and patent ductus arteriosus).

f. Know the history and physical examination findings characteristic of hypertrophic cardiomyopathy (HCM).

6. Sudden cardiac death.

a. Know what sports and age ranges have the greatest incidences of SCD and what etiologies of SCD are more common at what age ranges.

b. Be aware of how nonobstructive HCM presents and how exercise can worsen the symptoms of HCM.

c. Be familiar with coronary anomalies associated with sudden death, including the most common anomaly.

d. Identify the most frequently identifiable pathogen associated with myocarditis.

e. Know the significance of arrhythmogenic right ventricular dysplasia.

f. Be familiar with long-QT syndrome.

g. Be familiar with commotio cordis.

h. Know the appropriate management by a sports medicine physician of commonly detected arrhythmias and syncope.

7. Pulmonary disorders and exercise.

a. Be familiar with exercise-induced asthma (EIA), its presentation, common triggers (including association with temperatures and humidity), and treatment.

b. Know the role of chronic asthma treatment in managing EIA.

c. Know the likelihood of exercises with high minute ventilation versus those with low minute ventilation to exacerbate EIA.

d. Be able to recognize the presentation and treatment of vocal cord dysfunction.

e. Understand the physiologic responses to exercise that occur in individuals with chronic obstructive pulmonary disease.

\section{Nutrition, Metabolism, and Endocrine Issues}

1. Energy metabolism.

a. Describe the concept of energy balance including total energy expenditure, resting energy expenditure, physical activity, and thermal effect of food.

b. List the components that derive the universal source of metabolic fuel in the body (adenosine triphosphate) and the 3 energy systems used in muscular activity.

c. Describe the effects of aging on energy expenditure (resting metabolic rate and thermic effect of foods).

d. Describe the typical order of fuel consumption and its impact on athletic performance.

2. Nutritional needs for athletes.

a. For each condition describe the nutritional requirements of carbohydrates, protein, fat, and micronutrient intake: 
i. General training.

ii. Endurance athletes.

ii. Ultra-endurance athletes.

3. Hydration and fluid replacement.

a. Define dehydration and the potential causes of dehydration. Identify the effects on effort, performance, and balance control.

b. Identify the best way to evaluate a hydration program.

c. Describe the amount of fluid intake recommended before, during, and after exercise.

d. Identify the fluid recommendations if exercise lasts more than 1 hour.

e. Explain the effects of aging, medications and medical conditions, such as diabetes mellitus, on hydration status.

$f$. Identify the effects of hydration on heat illness and the expected levels of dysfunction associated with declining hydration.

4. Nutrient timing.

a. Identify the three phases of nutrient timing, and the recommended ingestion of nutrients during each phase.

b. Describe the recommended doses and examples of carbohydrates and protein snacks to enhance recovery from vigorous or endurance and resistance exercises.

5. Selected nutritional issues.

a. Identify the optimal time and ideal glycemic index for a pre-exercise meal.

b. Define carbohydrate loading and identify differences in performance with carb loading versus highfat/low-carb diet.

c. Recognize the different forms of eating disorders and their impact on athletic performance, bone mineralization, and menstruation (see also the section on the female athlete triad, to follow).

d. Discuss the impact of low-calorie meal consumption on weight loss and the impact of starvation mode on desired weight loss.

\section{e. Diabetes.}

i. Discuss the impact of nutrition and exercise on diabetes mellitus, including risk of increased weight, hypoglycemia and hyperglycemia. Describe how the effects of nutrition impact type I diabetes versus type 2 diabetes.

ii. Discuss the effects of overeating associated with insulin receptor upregulation.

iii. Discuss the effects of the glycemic index of foods on the diabetic athlete.

\section{f. Fractures.}

i. Discuss the nutritional risk factors that inhibit bone healing.

ii. Understand the role of nutrition in fracture management and recommended doses of calcium and vitamin $\mathrm{D}$ to promote bone healing.

iii. Differentiate the recommended doses of calcium for women based on their age/menopausal state. g. Distinguish between nonfunctional overreaching and overtraining syndrome.

i. Identify the clinical and hormonal signs and symptoms of each disorder.

ii. Identify possible markers for the detection of overtraining syndrome.

h. Discuss the impact of eating disorders on sport performance and effect on body systems.

6. Nutritional supplementation.

a. Identify the dose of creatine to maximize muscle mass and enhance energy and discuss the length of a typical washout period. Discuss the positive and negative effects of creating as it relates to athlete performance and weight gain.

b. Recognize the role and daily dose of caffeine in performance enhancement and potential side effects.

c. Know the uses and components of a sport drink versus an energy drink. Discuss the positive and negative aspects of these drinks as they relate to hydration, glucose, and sodium balance and carbohydrate, protein and fat metabolism.

d. Explain the risks associated with the use of nutritional supplements as they relate to drug testing in sport.

\section{E. Women's Health, Including Female Athlete Triad}

1. Differences between male and female athletes.

a. Describe the major differences in skeletal growth and development, physiology and body composition, and neuromuscular movement patterns in male and female athletes. Understand which differences remain despite correction for size.

b. Describe how these differences may affect training and performance.

c. Compare the prevalence and mechanisms of injury in female versus male athletes, including knee injuries (ACL injuries, PFPS), hip injuries (labral tears), joint hypermobility, and osteoarthritis.

2. The Female Athlete Triad and relative energy deficiency in sport (RED-F) [7].

a. Understand the definition of the triad, how it has evolved over the past 20 years, and the newer literature incorporating RED-F data, including how impaired physiological function alters metabolic rate, menstrual function, bone health, immunity, protein synthesis, and cardiovascular health.

b. Describe the interrelationship between nutritional status, reproductive function and bone metabolism. Describe risk factors including important historical questions to ask female athletes.

c. Nutrition: define energy availability and know how to calculate this for female athletes. Understand the nutritional spectrum from adequate energy availability to eating disorder.

d. Menstruation: understand the spectrum of menstrual dysfunction. Define primary and secondary 
amenorrhea, oligomenorrhea, luteal phase dysfunction and polycystic ovarian disease.

e. Bone metabolism: discuss the effect of different types of sports participation on bone accretion in the female athlete (with and without appropriate nutritional intake). Describe the difference between bone density and bone microarchitecture. Understand the utility of dual-energy X-ray absorptiometry and the difference between $T$ and $Z$ scores. Understand the pathophysiology of stress fractures in the setting of the female athlete triad.

f. Describe the appropriate clinical work-up for the female athlete who is suspected of having the triad/ RED.

g. Discuss the importance of interdisciplinary management. Understand the limitations of oral contraceptives in management athletes with this syndrome.

3. Exercise/activity in the peripartum female.

a. Discuss the maternal and fetal benefits and risks of exercise during pregnancy.

b. Describe the relative and absolute contraindications for exercise during pregnancy.

c. Understand the musculoskeletal changes that occur during pregnancy and postpartum phases.

d. Understand the limitations in imaging and medication management in pregnant and nursing females.

4. Pelvic floor dysfunction in the female athlete.

a. Describe the origin, insertion, and function of the muscles that comprise the pelvic floor.

b. Understand the types of pelvic floor dysfunction that occur in athletes.

c. Define stress urinary incontinence and associated risk factors.

d. Describe the clinical assessment and management of pelvic floor dysfunction in the female athlete.

\section{F. Pediatric Sports Medicine}

\section{Fractures}

a. Define diaphysis, metaphysis, epiphyseal plate, and epiphysis. Review the Salter-Harris Classification and explain which type of Salter-Harris fractures require surgical fixation. Explain the differences between buckle/ torus, greenstick, and plastic deformation fractures.

b. Define avulsion injury. Review the most common type of apophyseal injuries in the shoulder, elbow, pelvis, knee, and foot.

c. Explain why avulsion fractures are more common in the skeletally immature versus the skeletally mature. Review the common avulsion injuries at the anterior superior iliac spine, anterior inferior iliac spine, ischial tuberosity, and lesser trochanter.

d. Describe the natural history of slipped capital femoral epiphysis and Perthes disease, include clinical and radiographic findings as well as demographics.

2. Epiphysitis/overuse.

a. Define Little League shoulder and elbow. Explain the association between fatigue, pain, and throwing.
Review the pitching cycle and when injuries occur in each phase of the cycle.

b. Recognize the most common traction injuries to the pelvis, knee, ankle, and foot. Describe the natural history and contributing factors to these overuse injuries.

c. Explain the differences between spondylolisthesis and spondylolysis. Review the imaging studies used to diagnose pars interarticularis fractures. Review when to brace versus not brace with a pars fracture.

3. Osteochondrosis and osteonecrosis.

a. Define OCD. Review what makes a bony fragment stable versus unstable. State when to refer an OCD to a surgeon versus when to manage an OCD nonsurgically. Explain the difference between OCD and juvenile OCD. Review the most common locations for an OCD lesion in the knee and the ankle.

b. Define osteonecrosis. Identify and name the osteonecrosis lesions of the elbow, wrist, spine, hip, knee, ankle, and foot in the skeletally immature. For each lesion, describe the natural history, common demographic association, imaging modality, and treatment of choice.

4. Prevention and development.

a. Explain the importance of not specializing in one sport at an early age. Describe why appropriate attire, footwear, and appropriate fitting protective equipment are important to prevent injuries.

a. Discuss "love of sport" and why coaches and parents must not cause undue pressure to have children perform while injured.

c. Review the theories behind ACL injury in adolescents and the preponderance of female $A C L$ injuries. Analyze preventative strategies including prehabilitation programs designed to correct biomechanical risk factors and neuromuscular controls.

d. Describe why physiological differences between younger and older adolescent athletes can predispose these individuals to different injuries. State the importance of staying active and healthy and its effect on socialization, physical development, and maturation of our younger athletes.

5. Thermoregulation.

a. Review why greater surface area to body mass ration in children predisposes them to heat intolerance, including a slower acclimation rate.

b. Review fluid replacement guidelines before, during, and after exercise/competition for children and adolescents. Review the latest research on the importance of water, electrolyte-sports drinks, and chocolate milk as recovery drinks after competition in this age group.

\section{G. The Aging Athlete}

1. Reinforce to patients that regular exercise can increase life expectancy by limiting the development of chronic disease and disability [8]. Describe the natural physiological changes in the following systems: 
a. Cardiac: cardiac output, stroke volume, maximum heart rate. Review the contraindications to exercise specifically related to cardiac disease.

b. Pulmonary. Vital capacity: describe how quality of life, function, and fatigue are affected with endurance training in individuals with chronic obstructive pulmonary disease. Explain that necessity for maintaining activity to minimize the reduction of oxygen transport as we age due to inactivity [9].

c. Musculoskeletal system. Describe why resistance training may decrease the rate of loss of muscle mass as one ages. Explain why the risk of soft tissue injury increases due to changes in tissue compliance, flexibility, and stiffness. Describe the physiological changes that occur with osseous structures.

d. Describe how osteoarthritis can decrease exercise performance and how pain plays a role. Review the contraindications to exercise specifically related to osteoarthritis.

e. Athletics, exercise, and arthroplasty. Be able to discuss return to play and exercise guidelines based upon the prosthetic joint. Clarify that high-impact activities and twisting can cause breakdown of prosthetic joint.

f. Exercise prescription. Be able to prescribe the appropriate exercise prescription. Be up to date with standard recommendations of in a minimum of $150 \mathrm{mi}-$ nutes of moderate-intensity or 60 minutes of vigorous physical activity per week as long as there is no contraindication from a medical standpoint. Reiterate the importance of no more than a $10 \%$ increase in volume or intensity per week to minimize adverse events [9]. Prescribe balance activities as part of an exercise prescription for individuals with fall risks.

g. Benefits of exercise. Reiterate that exercise results in a decrease in all-cause mortality as well as cardiovascular disease, stroke, hypertension, type 2 diabetes mellitus, various forms of cancer (colon and breast) osteoporosis, obesity, cognitive impairment, anxiety, and depression [8]. Explain that a combination of resistance and aerobic training is the most effective form of exercise [8]. Describe how exercise affects quality of life and appreciate exercise is associated with improvements in overall psychological health, cognitive health, and well-being [8].

\section{H. Electrodiagnosis}

1. Nerve injury fundamentals. Describe the classification scheme for type and severity of nerve injuries (neurapraxia, axonotomesis, and neurotmesis). Associate the type of injury with the potential for recovery and prognosis and the expected electrodiagnostic findings (immediately postinjury, 4-6 weeks postinjury, months postinjury, and years postinjury). Describe indications and rationale for neurosurgical referral for a nerve injury as well as surgical treatment options. Explain the appropriate time course for the performance of the nerve conduction studies and needle electromyography examination for a suspect nerve injury and when repeat electrodiagnostic testing is indicated.

2. Specific nerve injuries. For each of the following nerve injuries, describe the typical clinical presentation, the affected nerve and specific location(s) of injury, the most common cause(s), and the electrodiagnostic findings specific to each injury.

a. Cervical spine: stinger, cervical radiculopathy, thoracic outlet syndrome, long thoracic neuropathy.

b. Shoulder: axillary neuropathy, suprascapular neuropathy, brachial plexopathy including ParsonageTurner syndrome.

c. Elbow/arm: Cubital tunnel syndrome; PIN syndrome and radial tunnel syndrome; AIN syndrome and pronator syndrome.

d. Hand/wrist: CTS, Guyon's canal UNE (aka cyclist's palsy). Contrast electrodiagnostic findings in an ulnar neuropathy at the wrist with an entrapment at the elbow. Explain the anatomic causation between wheelchair sports and CTS and ulnar neuropathy at the wrist.

e. Lumbosacral spine: lumbar radiculopathy. Sciatic neuropathy, lumbosacral plexopathy.

f. Hip/pelvis: pudendal nerve entrapment, with bicycle seat neuropathy (BSN). Femoral neuropathy, obturator neuropathy.

g. Knee: peroneal neuropathy. Explain the importance of EMG evaluation the head of the biceps femoris.

h. Foot/ankle: tarsal tunnel syndrome (TTS). List the 5 categories of TTS and describe the sensory and motor deficits in TTS. Name 3 neurologic syndromes that may produce foot drop.

3. Describe the evaluation and management of the following injuries:

a. Upper extremity compartment syndrome. Review the typical history, physical examination findings, and initial treatment of upper extremity compartment syndrome. Describe the pathophysiology and management of the of Volkmann's ischemic contractures.

b. Lower extremity compartment syndrome.

i. Acute compartment syndrome. Review the typical history, physical examination findings, and initial treatment of acute compartment syndrome.

ii. Chronic exertional compartment syndrome (CECS). Describe the natural history and treatment of CECS. Describe the compartments of the lower leg, including the blood supply, muscles and innervation within each compartment. Explain which athletes are most commonly affected by CECS.

c. BSN: Explain the natural history of BSN in cyclists and how a bicycle seat can be altered to improve symptoms.

\section{Prosthetics and Orthotics in Sports}

1. Perform an H\&P and demonstrate familiarity with upper and lower extremity amputations. Become 
familiar with common orthoses and prostheses, including proper technique for donning and doffing, cost, indications, and its effect on function.

2. Be familiar with each general topic below. Additional specific objectives also are listed.

a. Prosthetic fitting for athletes with an amputation. Describe the necessary components for a prosthetic prescription. Describe the $\mathrm{K}$ levels and prosthetic goals for patients at each $\mathrm{K}$ level. Describe the components of upper extremity and lower extremity prostheses. Describe special prosthetic considerations for athletes with lower extremity amputations who are active in swimming, fishing, golf, running, and/or skiing. Describe special considerations that need to be addressed during preparticipation physicals for athletes with an amputation.

b. Skin breakdown in athletes with an amputation. Describe the most common sites of skin breakdown among athletes with an amputation. Describe the pathophysiology of choke syndrome (verrucous hyperplasia) and how to manage/treat it. Describe the stages of skin breakdown. Describe prevention strategies and treatment for athletes with an amputation with skin breakdown, including diet recommendations and activity modification/sports participation.

c. Musculoskeletal injuries in athletes with an amputation. Describe the site(s) of peripheral nerve entrapment most common among athletes with a lower extremity amputation. Describe the pathophysiology, diagnosis, and treatment of neuromas. Contrast the common overuse injuries among active athletes with an upper extremity amputation compared with active athletes with a lower extremity amputation. Describe strategies for injury prevention, including training considerations, diet, and equipment/prosthetic fitting.

3. For each general category of orthoses, describe the indications and cost of commonly used types.

a. Shoulder orthoses, including sling and swaths, shoulder immobilizer, and clavicle strap.

b. Hand/finger orthoses, including thumb spica orthosis, neutral wrist orthosis, and ulnar gutters orthosis.

c. Knee orthoses, including a neoprene knee sleeve, patellar tracking orthosis, stabilizing knee orthosis, and off-loading knee orthosis.

d. Ankle orthoses, including a functional ankle brace (such as ankle stabilizing orthosis), stir-up ankle orthosis (such as air-cast), and compression ankle sleeve.

e. Foot orthoses, including a metatarsal pad, heel lift, medial or lateral heel wedge, and carbon fiber, and steel shank insert.

\section{J. The Disabled Athlete}

1. Perform H\&P and demonstrate familiarity with pathophysiology and equipment needs for patients with spinal cord injuries, amputations, and developmental disorders.
2. For each area to follow, be familiar with the general topic. Additional specific objectives follow.

a. PPE. Describe the recommended setting for performing a PPE for disabled athletes. Describe PPE considerations specific for disabled athletes. Demonstrate familiarity with screening tests and specific restrictions for athletes with Down syndrome.

b. Paralympics. Demonstrate familiarity with the various sports comprising the Paralympics, the athlete disabilities allowed to participate, and the equipment used in each of these sports.

c. Special Olympics. Demonstrate familiarity with the various sports comprising the Special Olympics and the athlete disabilities allowed to participate in them.

d. Autonomic dysreflexia. Describe the pathophysiology, symptoms treatment, and prevention of autonomic dysreflexia. Describe "boosting" and how it is used by athletes with spinal cord injury in competitive sports and how this is monitored and detected during competition.

e. Orthostatic hypotension. Describe the pathophysiology, symptoms, treatment, and prevention of orthostatic hypotension in patients with spinal cord injury.

f. Thermoregulation. Describe the pathophysiology, symptoms, treatment, and prevention of heat illness and hypothermia in patients with spinal cord injury.

g. Acute and chronic injuries. Describe traumatic and overuse injuries that are common among disabled athletes, including prevention and treatment.

i. Shoulder: Describe the rationale for higher injury rates in athletes with spinal cord injury and the specific conditions that are most typical.

ii. Elbow: Describe the rationale for higher injury rates in athletes with spinal cord injury and the specific conditions that are most typical.

iii. Hand/wrist/digits: Describe the rationale for higher injury rates in athletes with spinal cord injury and the specific conditions that are most typical.

iv. Fractures: Describe the rationale for higher injury rates in athletes with spinal cord injury and the specific areas that are the most affected.

h. Skin breakdown. Describe risk factors in athletes with spinal cord injury for skin breakdown and prevention strategies. Be familiar with staging and treatment of skin ulcers.

i. Peripheral nerve entrapments. Describe common sites for compressive mononeuropathies in wheelchair athletes. Be familiar with treatment and prevention for peripheral nerve injuries.

j. Osteoporosis. Describe what populations of disabled athletes are susceptible to osteoporosis and the related pathophysiology. Describe diagnostic workup, including laboratory testing and imaging, and treatment of osteoporosis in this population. Describe role of imaging to evaluate for fracture in the patient with osteoporosis. 
k. Visually impaired athlete. Be familiar with the sports in which visually impaired athletes can compete and the equipment required for competition. Discuss the interaction required with nonvisually impaired athletes working with the visually impaired.

\section{K. Exercise as Medicine and Integrative Sports Medicine}

1. Describe the role of the physician in promoting physical activity and demonstrate the ability to write an appropriate exercise prescription for individuals across the spectrum of age, ability, and wellness [10].

2. For each integrative sports medicine approach, describe its theory and principles of practice as well as its commonly employed methods/techniques. Know the conditions it is commonly used to treat and identify the clinical evidence for its efficacy, where available. Identify contraindications to its use. Additional objectives follow:

a. Acupuncture. Describe the differences between traditional versus contemporary acupuncture in terms of its goal and techniques.

b. Shock wave therapy. Understand the basic science of this modality.

c. Homeopathy and herbal remedies. Especially note those remedies that have been the most widely studied, as well as those that might be used for performance enhancement. Discuss the role of the naturopathic practitioner in sports and rehabilitation medicine.

d. Chiropractic/osteopathic. Describe the most common methods and their possible complications.

e. Massage therapy. Know the different types of massage therapy.

f. Alexander technique. Describe the role of the Alexander teacher and a basic lesson in this technique.

g. Yoga. Delineate the most popularly practiced types of yoga. Describe how the Eastern aspects of yoga distinguish it from Western athletic and rehabilitation approaches. Know possible complications of yoga.

h. Tai chi/chi gong. Describe the theories behind these practices as they relate to exercise and mental health.

i. Feldenkrais method. Describe a typical lesson.

j. Rolfing. Discuss how this method specifically targets the fascia, and describe the similarities and differences to massage therapy and traditional physical therapy.

\section{Eating Disorders in Athletes}

1. Prevalence. Understand that though eating disorders are more common in women than in men (10:1), both sexes are at risk. Recognize that eating disorders are more prevalent among athletes in general, and specifically in athletes participating in leanness (gymnastics, dance) sports or sports where weight classes are used (such as wrestling and rowing).

a. Diagnosis. Be familiar with the diagnostic criteria for anorexia nervosa, bulimia nervosa, binge eating disorder, other specified feeding or eating disorder, and other specified feeding or eating as outlined in the Diagnostic and Statistical Manual of Mental Disorders (DSM-V) criteria. Realize that many athletes have disordered eating, but not an eating disorder. Know how to use the PPE monograph and Female Athlete Triad Coalition questionnaire as screening tools. Describe the appropriate labs, review of system questions, and physical exam findings that may be present in the setting of an eating disorder.

b. Treatment. Describe the different types of treatment facility options (outpatient, hospital, residential treatment center) and when each is appropriate. Identify the different types of healthcare professionals that compose the multidisciplinary team needed for the optimal treatment of a patient with an eating disorder.

c. Comorbidities and consequences. Identify the comorbid mental health disorders that often accompany eating disorders. Know how eating disorders can adversely affect gastrointestinal, skeletal, cardiac, reproductive and muscle health. Describe sport performance consequences of eating disorders.

d. Return to sport. Know the return-to-sport guidelines detailed in the Female Athlete Triad: Cumulative Risk Assessment in the 2014 Female Athlete Triad Coalition Consensus Statement on Treatment and Return to Play of the Female Athlete Triad [11].

e. Prevention. Describe what efforts can be made with athletes, coaches, athletic administrators, and parents to prevent eating disorders. Identify risk factors for eating disorders and how to discuss them with patients.

\section{Basic Science Foundations of Sports Medicine}

\section{A. Pathophysiology of Tissues and Organs}

Understand the basic organization of tissue types, with an emphasis on the aspects of the structural elements to follow. Know the effect of aging, training, medications, hormones, drugs, nutrition, and therapeutic interventions on these structural elements.

1. Skeletal muscle.

a. Muscle cell/fibers, including their types, arrangement/composition.

b. Muscle contraction, including action potentials, role of actin and myosin, calcium and acetylcholine.

c. Determinants of strength and response to training and disuse.

d. Strains, including types and healing rates.

2. Tendons.

a. Basic structure, including paratenon.

b. Blood supply and healing.

c. Collagen: types, arrangement, cross-linking.

d. Elastin: fibroblastic response; metabolic rate of tendon.

e. Golgi tendon organs.

3. Bone. 
a. Bone cells and their function, including osteocytes, osteoblasts, and osteoclasts.

b. Basic structure and function of cortical versus trabecular bone.

c. Blood supply.

d. Normal versus abnormal bone formation, including response to stress and inborn errors.

e. Growth plates.

4. Ligaments.

a. Basic composition, including blood supply.

b. Sprains: describe types and healing rates.

5. Nerves.

a. Neuronal structure, including axon versus dendrites.

b. Fascicular organization, including epineurium, perineurium, endoneurium.

c. Schwann cell.

d. Neuron type, including small versus large, myelinated versus unmyelinated.

e. Neuromuscular transmission: presynaptic versus postsynaptic, modulators of membrane potential.

f. Nerve cell injury, including neurapraxia, neurotmesis, axonotmesis.

g. Understand the effect of nerve injury on return to play, the role of electromyography/nerve conduction study findings, ultrasound versus MRI findings, bracing, protective devices, surgical versus nonsurgical considerations.

6. Fascia.

a. Basic composition.

b. Functional/clinical significance.

\section{B. Fundamental Biomechanical Principles}

1. General biomechanics.

a. Compare and contrast isometric versus isotonic versus isokinetic resistance exercises and how they may be implemented in a rehabilitation program.

b. Describe the difference between concentric and eccentric muscle contractions.

c. Discuss differences between "open" versus "closed" kinetic chain exercises, give examples of each, and the indication/role for each type of exercise.

d. Compare and contrast passive, active assisted, and active range of motion.

e. Compare and contrast dynamic versus static stretching. Review the most appropriate time to stretch, pre- or postexercise.

2. Lower Limb Biomechanics.

a. Describe the motion planes (sagittal, coronal, and transverse) and range of motion for each of the following joints: hip, knee, ankle, foot, and first MTP.

b. Describe the ideal neutral stance position, including alignment of the weight-bearing line through the lower limb.

c. Describe biomechanical differences between walking and running, including foot contact, stance and swing phase, vertical ground reaction force, and joint motion excursions. d. Explain differences between heel strike versus forefoot strike running patterns, including clinical implications of each.

e. Outline how to counsel patients regarding footwear, including fit, general structure, motion control properties, cushioning, and wear patterns.

f. Describe how suboptimal lower limb biomechanics may lead to specific injuries, including plantar fasciitis, Achilles tendinopathy, lateral ankle sprain, medial tibial stress syndrome, patellar tendinopathy, patellofemoral syndrome and stress fractures.

g. Describe how biomechanical abnormalities may be managed with orthotics, taping, or bracing.

h. Describe the biomechanical differences that may predispose female athletes to noncontact anterior cruciate ligament injuries and how teaching appropriate jumping/landing techniques may prevent them.

i. Describe the biomechanics of cycling, including proper seat height/position for the cyclist and how improper height/position can lead to injury.

3. Upper limb biomechanics.

a. Describe the motion planes (sagittal, coronal and transverse) and range of motion for each of the following joints: shoulder, elbow, wrist, and hand.

b. Describe the phases of throwing with involved muscle activity, including wind-up, early cocking, late cocking, acceleration, deceleration, and followthough.

c. Describe the kinetic chain of throwing, including contributions from the legs, trunk, shoulder girdle, and upper limb.

d. Describe common biomechanical abnormalities demonstrated in pitching and how these may lead to injuries of the shoulder and elbow. Describe which types of injuries most commonly occur in each phase.

e. Describe common technical errors in specific swimming strokes that are thought to be risk factors for shoulder injury.

\section{Imaging Fundamentals}

1. Approach. Describe a general approach to imaging, eg, how generally the choice of imaging is influenced by the history and physical exam, and how the results will be integrated into an assessment and plan.

2. For each of the diagnostic imaging modalities to follow, describe how it works, general category of use, advantages and disadvantages, and contraindications. Additional learning objectives follow:

a. Plain radiographs. Describe the characteristics of a fracture on radiograph. Describe how many radiograph views are required for fracture identification and why. Delineate the appearance of a degenerative joint. Discuss the appearance (or lack) of the major visible structures on radiographs, including, but not limited to air, fluid, bone, cartilage, muscle, calcific deposits, and the major visible organs. Understand the role of fluoroscopy in spinal interventions. 
b. Computed tomography. Discuss the role of $\mathrm{CT}$ in evaluation of injuries-which are likely to benefit from the use of CT as opposed to MRI. Discuss when contrast should be used in conjunction with $\mathrm{CT}$ imaging. Identify circumstances when 3D reconstruction would be most helpful. Describe the indications for CT angiography in sports medicine. Delineate the findings of acute intracranial hemorrhage, intra-abdominal visceral injury, and bone healing. Discuss the advantages and disadvantages of using $\mathrm{CT}$ for needle guidance.

c. Magnetic resonance imaging. Understand the difference between the different sequences, including T1-weighted, T2-weighted, proton density, and STIR (ie, short tau inversion recovery)-specifically state how cortical bone, medullary bone, cartilage, tendon, muscle, and fluid should appear. Be able to recognize the appearance of tendinopathy, fracture, cartilage injury and fluid collections. Identify the circumstances in which MRI provides unique and clinically critical information. Describe when contrast should be used intra-articularly and intravascularly. Describe MR neurography.

d. Musculoskeletal ultrasound $[12,13]$. Identify the characteristic appearance of different tissue types in this modality. Describe the advantages and disadvantages of this modality, including resolution, portability, cost, and accessibility. Describe sono-palpation. See separate section on musculoskeletal ultrasound for more details.

e. Bone scintigraphy. Describe the 3 phases of the test. Understand the role in sports medicine centered on detection of stress injury to assess healing. Understand the use of SPECT (ie, single-photon emission computed tomography) in conjunction with this imaging modality.

f. Cardiovascular/vascular imaging.

i. Echocardiogram indications in athlete.

ii. Doppler ultrasonography.

iii. Contrast angiography, venography, and arteriography (positional).

\section{Laboratory Studies}

1. Demonstrate an understanding of the correlation and pertinence of routine and specialized laboratory tests with regard to various injuries and sport specific illnesses that a team physician may need to order or encounter under the following circumstances.

a. Preparticipation, including routine blood work, genetic testing, anemia-related tests.

b. Endurance events, including electrolyte panel, sweat and urine tests, and blood tests for rhabdomyolysis.

c. Cardiovascular assessment, including genetic markers of cardiac disease.

d. Pulmonary, including pulmonary function tests, and methacholine challenge test.

e. Hematologic disorders, including sickle cell trait/disease, clotting factor deficiencies, G6-PD hereditary spherocytosis, March hemoglobinuria. f. Infection, including hepatitis, bacterial, HIV, herpes simplex virus, bite wounds, sexually transmitted diseases, heterophile antibody (monospot)/throat culture, Epstein-Barr virus-specific antibody; HIV testing, including legal requirements.

g. Diabetes, including hemoglobin A1c, glycosylated hemoglobin, glucose tolerance test, casual plasma glucose versus fasting, and routine laboratory test.

h. Other endocrine considerations, including pregnancy test, testosterone levels, gonadotropin-releasing hormone, follicle-stimulating hormone, luteinizing hormone (karyotypes) prolactin, thyroid-stimulating hormone, free thyroxine, and calcium progestinwithdrawal.

i. Understand tests for blood doping/banned substances/ergogenic aides, including Hematocrit levels, erythropoietin, electrolytes, liver function tests, lipid profile, human growth hormone, urinalysis, urine screens, uric acid, testosterone/epitestosterone ratio, gas chromatography/radioimmunoassay/mass spectrometry.

\section{E. Commonly Used Medications}

1. Familiarize yourself with the common banned substances in competitive sports, such as may be found on the National Collegiate Athletic Association (NCAA) Banned Drugs list, as well as those which require declarations of use rather than therapeutic use exemptions.

2. For each class of medication be familiar with mechanism of action, appropriate use, and common and severe adverse medication effects. Additional objectives follow.

a. Nonsteroidal anti-inflammatory drugs (NSAIDs).

i. Identify the primary mechanism of NSAIDs action for reduction of pain and inflammation.

ii. Name medications that can be administered in conjunction with NSAIDs to reduce the likelihood and severity of some of the common adverse effects associated with NSAID use.

iii. Describe a potentially severe electrolyte abnormality that can be associated with NSAID use.

iv. Describe the role of NSAID administration in association with muscle contusion.

v. Explain why NSAID use may be more appropriate during the acute phase of injury recovery but may be contraindicated in later stages.

vi. Describe the role of topical NSAIDs.

b. Injectables.

i. Understand the side-effects and risks associated with injection of corticosteroids.

ii. Know the role and efficacy of injectable corticosteroids in conditions typically encountered in sports medicine (eg, acute injury, osteoarthritis, chronic tendinopathy).

iii. Be aware of the different formulations and administration schedules available for injectable hyaluronic acid, as well as risks and benefits of each. 
iv. Be up to date on the evidence-based medicine research and appropriate usage regarding orthobiologics [14-18].

\section{c. Asthma medications.}

i. Be familiar with the sports that have the greatest prevalence of exercise-induced bronchospasm.

ii. Know the role of short- and long-acting $\beta$-agonists, as well as other classes of asthma medications (leukotriene receptor antagonists, mast cell stabilizers, etc) in the management of exercise-induced bronchospasm.

iii. Recognize common detrimental effects $\beta$ agonists can have on performance.

iv. Identify which inhaled $\beta$-agonist combinations are permitted by the NCAA versus those permitted by the World Anti-Doping Agency (WADA).

d. Cardiovascular medications.

i. Identify why diuretic agents should be avoided or used only with caution in athletes, explicating potential adverse side effects and their potential use for illicit purposes.

ii. Understand why $\beta$-blockers are prohibited in certain precision sports (eg, archery). Explain how $\beta$ blockers may adversely affect athletic performance (cardiac output, $\mathrm{VOZ}_{\max }$, cardiac output, fuel use, thermoregulation, skeletal muscle recruitment patterns) and understand the role of selective $\beta$ blockers.

iii. Given the potential problems with diuretics and $\beta$-blockers, suggest alternative anti-hypertensive medications that may be a better choice in athletes.

iv. Identify the most significant potential risk associated with using statins in athletes.

\section{e. Antibiotics.}

i. Understand the potential risks of prescribing fluoroquinolones for athletes.

ii. Know the first-line agents for treatment of community-acquired methicillin-resistant Staphylococcus aureus (CA-MRSA).

iii. Know how treatment of CA-MRSA can contribute to the development of infectious diarrhea.

iv. Be familiar with the role of decolonizing agents in the management of CA-MRSA.

\section{f. Stimulants.}

i. Be familiar with stimulant medications commonly used to treat attention-deficit/hyperactivity disorder (ADHD).

ii. Understand the advantages athletes may derive from stimulant medications and restrictions on their use as proscribed by the International Olympic Committee and the WADA.

g. Oral contraceptive pills (OCPs).

i. Understand the role of OCPs in the treatment of menstrual irregularities associated with the female athlete's triad.

ii. Be familiar with the role of OCPs in potentially reducing the risk of stress-fractures. iii. Know the potential severe adverse/lifethreatening side-effects associated with OCP use, as well as those which may affect performance.

h. Allergic rhinitis. rhinitis.

i. Identify first-line treatments for allergic

ii. Be aware of restrictions on medications such as pseudoephedrine and other over-the-counter treatments which athletes may use as self-treatment for symptoms associated with allergic-rhinitis.

3. Doping control.

a. Be familiar with the varying agencies (WADA, United States Anti-doping Agency, NCAA), which monitor and govern the use of illicit substances by athletes, including the sports-specific antidoping organizations.

b. Understand the requirements for a valid therapeutic use exemption.

c. Be aware if any restrictions regarding orthobiologics (eg, but not limited to platelet-rich plasma, stem cells, and bone marrow aspirate concentrate).

\section{Special Topics Within Sports Medicine}

\section{A. Preparticipation Physical Examinations (PPEs)}

Describe the contents of the standard PPE, particularly the historical questions related to cardiovascular risk $[6,19]$. Additional objectives follow.

1. Identify the goals of the PPE, including forming a therapeutic alliance with the athlete, and the different types of prevention.

2. Identify the major cardiovascular, pulmonary, medical, neurologic, and musculoskeletal injuries that can predispose to loss of life, limb, other morbidity, or diminished sports function should be sought first. Describe the diagnoses that disqualify an athlete from safe participation in contact sport.

3. Describe the cost and benefits of the different types of cardiac screening modalities.

4. Be familiar with what referral resources to which a PPE team should have access.

5. Describe the pros and cons of a station-based PPE.

6 . Know the most common problem areas for individual sports.

7. Identify resources for determining age-appropriate expectations for vital signs and functioning.

8. Describe resources to direct athletes for recommendations for performance improvement.

9. Be familiar with timing of need for repeating physical examinations.

10. Describe basic principles of return to play for previously injured athletes.

11. Be able to direct appropriate sports return based on previous injuries/surgeries.

12. Understand the best practice for concussion baseline testing at the high school collegiate and professional levels. 


\section{B. Sideline Coverage and Emergencies}

1. Staff responsibilities. Discuss the importance of the team physician knowing the affiliated staff (coach, athletic director/event director, athletic trainers, Emergency Medical Technicians, event security, other physicians) and their roles, as well as having a clearly recognized chain of command on and off the field.

2. Location and supplies. Articulate the importance of knowing the venue address, nearest hospital, floor plan/field layout, location of automated external defibrillator, spine board, ambulance, etc. Identify appropriate equipment kit.

3. Communication and protocols. Describe the role of established protocols for evaluation, treatment, and disposition of injuries, including an Emergency Action Plan. Discuss appropriate technology for communication among staff. Identify the importance of protocols for clearing injury area to protect the injured and treating staff, especially during races. Discuss the importance of protocol review and practice in event preparation.

4. Emergencies.

a. Know what the specific needs are among staff regarding Basic Life Support, Advanced Cardiovascular Life Support, and Advanced Trauma Life Support certification.

b. Describe the prioritization protocol in injuries referenced by the $A B C D E F$ acronym (Airway, Breathing, Circulation, Disability, Exposure, Final Disposition), and for each of the below types of injuries, discuss how to apply the ABCDEFs.

i. Head and neck injuries: mild traumatic brain injury, spinal cord injuries.

ii. Ear-nose-throat injuries.

iii. Cardiac events-sudden cardiac arrest.

iv. Thorax-pneumothorax, exercise-induced bronchospasm.

v. Abdominal, pelvic, and genitourinary injuries.

- Describe the most common areas of injury related to blunt abdominal trauma, the liver, spleen, and bladder [20]. Describe the most common location of pain after a liver injury due to blunt abdominal trauma and the associated referral pain pattern. Provide a guideline for return to play [21]. Describe the Kehr sign and the common presentation after a splenic injury. Provide postsplenectomy precautions. Provide a guideline of return-to-play guidelines after splenectomy [21]. Describe the common presentation for blunt bladder trauma, including inability or difficulty voiding, gross hematuria, suprapubic or abdominal pain [22].

- Return to play [21]. Be familiar with the literature and controversy that there is no consensus on return to play, including duration and intensity, after splenic and liver injury. Recommending light activity in the first 3 months after injury and increase activity level as symptoms allow is reasonable.

vii. Musculoskeletal and extremity injuries.

viii. Environmental and miscellaneous emergencies, including heat and cold exposure and anaphylaxis.

\section{Primary Care Sports Medicine Issues}

For the following body systems, be familiar with the common conditions listed below, including presentation, treatments, effect on athletic participation, and return-to-play policy.

1. Cardiac. Myocarditis, hypertension, hypertrophic cardiac myopathy, long Q-T syndrome, cardiac arrhythmias, cardiac murmurs, Marfan syndrome.

2. Respiratory. Dyspnea, asthma, exercise-induced bronchospasm, vocal cord dysfunction, exercise induced anaphylaxis.

3. Gastrointestinal. Acute gastroenteritis, peptic ulcer disease, delayed gastric emptying, runner's diarrhea, lower gastrointestinal bleeding, acute hepatitis, hepatosplenomegaly.

4. Endocrine. Diabetes, including type I and II; hypothyroidism, hyperthyroidism.

5. Renal and Genitourinary. Hematuria, proteinuria, acute renal failure, nephrolithiasis, stress incontinence, genital injuries, urinary tract infections, direct injuriestesticular trauma, penile trauma, vulva trauma.

6. Hematology/immunology. Sickle cell trait, venous thromboembolism, anemia: sports dilution, irondeficiency.

7. Infectious disease. Upper respiratory infections, infectious mononucleosis (note especially return-toplay guidelines), influenza, herpes simplex, sexually transmitted diseases (chlamydia, gonorrhea), blood borne pathogens (HIV, hepatitis $B$, hepatitis $C$ ).

\section{Sports Dermatology}

For each skin lesion, be able to accurately describe and identify. Additional objectives follow.

1. Environmental injuries. Describe appearance, treatment, and prevention of environmental injuries including, sunburn, frostnip/frostbite, and chilblains (perino).

2. Urticaria. Describe the treatment of the various types of urticaria, including acquired cold urticarial, solar urticaria, aquagenic urticaria, and exerciseinduced urticaria.

3. Skin infections. Describe the typical symptoms, examination findings, testing, pathogen, treatments, and restrictions from competition/practice (if any).

a. Impetigo, folliculitis.

b. MRSA, abscess, and furunculosis. Describe the specific procedure for performing an incision and drainage for the treatment of an abscess.

c. Cellulitis.

d. Scabies.

e. Molluscum contagiousum.

f. Herpes gladiatorum.

g. Tinea corporis/pedis.

h. Warts.

i. Swimmers' ear.

j. Swimmer's itch.

k. Seabather's eruption. 
4. Skin lacerations. Describe the initial wound management and evaluation of a skin laceration. Describe the specific procedure for suturing a laceration to the face/scalp and trunk. Discuss what suture material is recommended for trunk, extremity/scalp, and facial lacerations. Describe common suturing techniques and the indications for each. Describe the specific procedure for applying tissue adhesives (DERMABOND) to a laceration and any contraindications to this.

5. Auricular hematoma (Cauliflower ear). Describe the typical appearance, cause, and treatment.

\section{E. Musculoskeletal Ultrasound}

1. Be familiar with the American Institute of Ultrasound Medicine (AIUM) guidelines for musculoskeletal ultrasound found at: http://www.aium.org/resources/ guidelines/musculoskeletal.pdf [23].

\section{Basics principles.}

a. Understand the relative strengths and weaknesses of musculoskeletal ultrasound compared with other imaging modalities. Be able to discern appropriate circumstances for use of musculoskeletal ultrasound.

b. Be familiar with the basic principles of impedance, reflection, refraction, and absorption as it relates to musculoskeletal ultrasound.

c. Define echogenicity, and understand the descriptive terms anechoic, hypoechoic, and hyperechoic.

d. Understand anisotropy/the anisotropic effect, and how to account for it when using musculoskeletal ultrasound.

e. Understand other common ultrasound artifacts such as reverberation, acoustic shadowing, and through transmission.

f. Understand how frequency, resolution, and attenuation affect ultrasound imaging.

g. Be familiar with the basic knobs of the ultrasound machine and their functions.

h. Understand the role of adjusting depth, gain, focal zones, and time gain compensation on images obtained.

i. Understand the role of Doppler imaging in musculoskeletal ultrasound, including their role in assessing neovascularization.

3. Basic technique.

a. Familiarize yourself with how to make appropriate transducer selection.

b. Know the role of ultrasound gel.

c. Recognize the importance of anchoring the hand during scanning.

d. Be familiar with conventions of transducer positioning (ie, left and right on screen in relation to transducer orientation and anatomic positions such as proximal, distal, medial, and (ateral).

4. Imaging.

a. Familiarize yourself with the normal ultrasonographic appearance of typical structures encountered in musculoskeletal ultrasound (eg, muscle, tendon, ligament, nerve, vessels, and bone). b. Be able to scan and obtain images for the 6 major regions of the body as recommended by the AIUM Practice Guidelines for musculoskeletal ultrasound (shoulder, elbow, wrist/hand, hip, knee, foot/ ankle) [23].

5. Intervention.

a. Be familiar with the principles and techniques of injection and other interventions commonly performed in a sports medicine office.

i. Understand the different techniques involved in needle guidance in-plane versus out-of-plane relative to the transducer.

ii. Perform basic interventional injections to areas that require ultrasound guidance for correct placement (ie, hip joint, biceps tendon sheath, glenohumeral joint, etc).

\section{F. Communication and Teamwork}

Understand the following important topics/ principles.

1. General communication. Describe common pitfalls associated with improper and adverse forms of communication. Understand the importance of setting "on-call" expectations with your certified athletic trainer and setting consistent times for training room sessions. Learn that it is the responsibility of the team physician to blend into the Training Room Environment.

2. Availability. Discuss the importance of physician availability. Identify the sports with greatest risks of injury. Learn to triage communication in an effective, accurate, and efficient manner.

3. Teamwork. Understand the importance of developing relationships with the team's certified athletic trainers, head coaches, and any other medical personnel that may assist in treating your athletes.

4. Emergencies. Describe the important elements of an EAP, including how they might be different for different venues. Please see the section on Sideline Coverage and Sports Medicine Emergencies.

5. Chain of command. Describe the importance of having a predetermined chain of command, including a single person who will be the voice of the medical staff. Appreciate that the head certified athletic trainer is the liaison between the head coach and the physician. Explain why communication from the head team physician to the director of athletics (at the collegiate level) can be considered reasonable if an emergency situation occurs. Know that emotions are heightened during games, and protocols are established during preseason planning to avoid miscommunication.

6. Media. Understand the role of the team physician regarding relations with the media and the mechanism for communicating information. Recognize the importance of speaking with "one voice" as medical providers. Understand that social media has changed the speed at which information can be delivered. 


\section{G. Ethical and Medico-Legal Aspects and}

Professionalism Serving as Team Physician

Understand the following important topics/

principles.

1. Advocacy. Explain the role of the team physician, specifically identifying for whom she is an advocate.

2. Autonomy. Describe the role of the team physician in providing informed consent to the athlete and the importance in making decisions in the context of the player's personal goals and preferences.

3. Liability. Understand the importance of limiting unnecessary liability for the team physician or the institution. Describe why a physician may or may not be covered to take care of her student athletes when they are out of state, and identify resources to check individual state rules. Identify what rules govern the liability of physicians caring for other teams' players, spectators, or other staff members.

4. Confidentiality. Describe HIPPAA (the Health Insurance Portability and Accountability Act of 1996) and FERPA (the Family Educational Rights and Privacy Act of 1974). Understand how the physician's relationship with the school or professional club inhibits the normal strict rules governing doctor-patient confidentiality. Describe how the physician's responsibility regarding giving players advance notice of any potential sharing of medical information.

5. Minors. Describe when and how parents should be involved in their child's training and care and when parental permission needs to be obtained.

6. Professionalism and ethics. Describe the important qualities of a team physician, including altruism, honesty, compassion, integrity, and respect. Describe the physician's responsibility to self, patient, institution, and medical profession.

7. Prevention. Discuss the role of the PPE (see the previous section on Screening and Prevention).

8. Infectious disease. Describe the role of the physician in limiting the spread of infectious diseases (see Primary Care Sports Medicine section above).

9. Drugs and the athlete (see the previous section, "Commonly Used Medications").

\section{H. Mental Health and the Athlete}

1. Introduction: Be aware that mental illness remains a major public health concern affecting broad segments of the population, including athletes of all ages. Due to the lack of mandatory or consistent reporting systemic it's likely that mental health concerns are underreported in the athletic population [24,25].

2. Depression.

a. Be familiar with the diagnostic criteria for depression, as defined by the DSM-V, including 5 or more major criteria persisting more than 2 weeks, including (but not limited to) diminished interest in activity, weight change, sleep disturbance, fatigue, difficulty concentrating, and recurrent thoughts of death. b. These symptoms must cause clinically significant distress or impairment in social, occupational, or athletic function and may not be attributable to another illness or condition.

c. Discuss the incidence of depression in the athletic population and how it compares with the general population of comparable age.

d. Review commonly used screening tools for depression $[26,27]$.

e. Describe the unique risk factors experienced by athletes that could impact their mental health.

f. Consider the differential diagnosis for depression in the athlete (circumstantial, injury time loss, dysthymia, bipolar disorder, cluster B personality traits, seasonal affective disorder, premenstrual dysphoric disorder, substance abuse, and comorbid illness).

g. Discuss the demographic risk factors for depression, including age, race, sex, ethnicity, and level of competition.

3. Anxiety.

a. Be familiar with the DSM-V criteria for generalized anxiety disorder and other anxiety related illness. Typically, diagnosis is considered for symptoms occurring more days than not for greater than 6 months duration related to a number of activities and is difficulty to control.

b. Discuss how the diagnosis of anxiety is different in youth versus adult athletes.

c. Discuss the conditions that may provoke anxiety (both acute and chronic) in athletes.

d. Review screening tools to identify anxiety in athletes.

e. Discuss biofeedback and cognitive behavioral therapy technique for stress and anxiety reduction.

4. Suicide.

a. Discuss the incidence of suicide in athletes at the (1) high school, (2) collegiate, and (3) professional level.

b. Discuss the changes in factor into clarifying the true incidence and prevalence of suicide in the athletic population.

c. Discuss what diagnoses seen in athletes make them more vulnerable to suicide (eating disorder, PTSD, schizophrenia).

d. Discuss strategies to manage suicide both acutely and via a preventative approach.

e. Discuss the community impact of athletic suicide.

5. Attention deficit disorders.

a. Discuss the diagnostic criteria required for diagnosis of $A D H D$ in children ( $<18$ years old) versus adults ( $>18$ years old).

b. Discuss the changes to the ADHD criteria made between DSM-IV and DSM-V and how this pertains to diagnosis and management of attention deficit.

c. Consider the types and situations in which an athlete may prompt an evaluation for attention deficit disorder (inattention, hyperactivity, impulsivity, defiance hostility, etc). 


\section{d. Discuss the different types of attention deficit disorders.}

e. Consider the differential diagnosis for attention deficit disorders (bipolar disorder, substance abuse, etc).

6. Bullying and hazing.

a. Definite bullying and hazing.

b. Consider the challenge of identifying hazing in the athletic culture.

behaviors.

c. Discuss strategies to reduce or eliminate

d. Consider the impact of bullying and hazing on the mental health of the impacted athlete and team culture.

7. Eating Disorders

Athletes."

a. Please refer to the section "Eating Disorders in

\section{References}

1. Asif IM, Stovak M, Ray T, Weiss-Kelly A. Primary Care Sports Medicine Fellowship: AMSSM Proposed Standards of Excellence. Clin J Sport Med Off J Can Acad Sport Med 2017;27:231-244.

2. Accreditation Council for Graduate Medical Education. Physical Medicine and Rehabilitation. Available at http://www.acgme.org/ Specialties/Overview/pfcatid/17. Published March 25, 2017. Accessed March 25, 2017.

3. McCrory P, Meeuwisse W, Dvorak J, et al. Consensus statement on concussion in sport-the 5 (th) international conference on concussion in sport held in Berlin, October 2016. Br J Sports Med, in press.

4. Leddy J, Hinds A, Sirica D, Willer B. The role of controlled exercise in concussion management. PM R 2016;8(3 Suppl):S91-S100.

5. Leddy JJ, Baker JG, Willer B. Active rehabilitation of concussion and post-concussion syndrome. Phys Med Rehabil Clin N Am 2016; 27:437-454.

6. Drezner JA, Sharma S, Baggish A, et al. International criteria for electrocardiographic interpretation in athletes. $\mathrm{Br} \mathrm{J}$ Sports Med 2017;51:704-731.

7. Mountjoy M, Sundgot-Borgen J, Burke L, et al. The IOC consensus statement: Beyond the Female Athlete Triad-Relative Energy Deficiency in Sport (RED-S). Br J Sports Med 2014;48:491-497.

8. American College of Sports MedicineChodzko-Zajko WJ, Proctor DN, et al. American College of Sports Medicine position stand. Exercise and physical activity for older adults. Med Sci Sports Exerc 2009;41:1510-1530.

9. Concannon LG, Grierson MJ, Harrast MA. Exercise in the older adult: From the sedentary elderly to the masters athlete. PM R 2012;4:833-839.

10. ACSM. ACSM | EIM. Exercise is Medicine ACSM Initiative. Available at http://www.acsm.org/about-acsm/initiatives/eim. Accessed March 25, 2017.
11. De Souza MJ, Nattiv A, Joy E, et al. 2014 Female Athlete Triad Coalition consensus statement on treatment and return to play of the female athlete triad: 1st International Conference held in San Francisco, CA, May 2012, and 2nd International Conference held in Indianapolis, IN, May 2013. Clin J Sport Med 2014;24: 96-119.

12. Finnoff JT. The evolution of diagnostic and interventional ultrasound in sports medicine. PM R 2016;8(3 Suppl):S133-S138.

13. Finnoff JT, Hall MM, Adams E, et al. American Medical Society for Sports Medicine position statement: Interventional musculoskeletal ultrasound in sports medicine. Clin J Sport Med 2015;25:6-22.

14. Ajibade DA, Vance DD, Hare JM, Kaplan LD, Lesniak BP. Emerging applications of stem cell and regenerative medicine to sports injuries. Orthop J Sports Med 2014;2. 2325967113519935.

15. Best TM, Caplan A, Coleman M, et al. Not missing the future: A call to action for investigating the role of regenerative medicine therapies in pediatric/adolescent sports injuries. Curr Sports Med Rep 2017;16:202-210.

16. Hogan MV, Walker GN, Cui LR, Fu FH, Huard J. The role of stem cells and tissue engineering in orthopaedic sports medicine: Current evidence and future directions. Arthroscopy 2015;31:10171021.

17. Malanga G, Nakamura R, Nakamurra R. The role of regenerative medicine in the treatment of sports injuries. Phys Med Rehabil Clin N Am 2014;25:881-895.

18. Sepúlveda F, Baerga L, Micheo W. The role of physiatry in regenerative medicine: The past, the present, and future challenges. PM R 2015;7(4 Suppl):S76-S80.

19. Drezner JA, O'Connor FG, Harmon KG, et al. AMSSM Position Statement on Cardiovascular Preparticipation Screening in Athletes: Current evidence, knowledge gaps, recommendations and future directions. $\mathrm{Br} \mathrm{J}$ Sports Med, in press.

20. Rifat SF, Gilvydis RP. Blunt abdominal trauma in sports. Curr Sports Med Rep 2003;2:93-97.

21. Juyia RF, Kerr HA. Return to play after liver and spleen trauma. Sports Health 2014;6:239-245.

22. Guttmann I, Kerr HA. Blunt bladder injury. Clin Sports Med 2013; 32:239-246.

23. AIUM. The American Institute of Ultrasound in Medicine (AIUM). AIUM Musculoskeletal Ultrasound Examination. Available at http://www.aium.org/resources/guidelines/musculoskeletal.pdf. Published March 25, 2017. Accessed March 25, 2017.

24. Rao AL, Hong ES. Understanding depression and suicide in college athletes: Emerging concepts and future directions. Br J Sports Med 2016;50:136-137.

25. Centers for Disease Control and Prevention. National Center for Health Statistics on Dementia and Mental Health. National center for Health Statistics. Available at http://www.cdc.gov/nchs/ fastats/depression.htm. Accessed May 22, 2017.

26. Kroenke K, Spitzer RL, Williams JBW. The PHQ-9. J Gen Intern Med 2001;16:606-613.

27. Spitzer RL, Kroenke K, Williams JBW, Löwe B. A brief measure for assessing generalized anxiety disorder: The GAD-7. Arch Intern Med 2006;166:1092-1097.

\section{Disclosure}

J.L.Z. Adolescent and High School Sports Medicine Outreach Program, Department of Orthopedics and Rehabilitation, Divisions of PM\&R, Sports Medicine, \& Research, University of Florida Orthopaedics and Sports Medicine Institute (OSMI), PO Box 112727, Gainesville, FL 32611. Address correspondence to J.L.Z.; e-mail: zaremjl@ortho.ufl.edu

Disclosure: nothing to disclose

M.C.D. Rusk Institute of Rehabilitation Medicine, New York University School of Medicine, New York, NY

Disclosure: nothing to disclose
A.A. Department of Physical Medicine and Rehabilitation, University of Michigan Hospital and Health Systems, Michigan NeuroSport Program, Sports Medicine and Rehabilitation, Eastern Michigan Team Physician, Ann Arbor, MI Disclosure: nothing to disclose

E.C. Penn Center for the Female Athlete, Penn Musculoskeletal Center, University of Pennsylvania, Philadelphia, PA

Disclosure: nothing to disclose 
B.D. Department of PM\&R, University of California, Davis Medical Center, Sacramento, CA

Disclosure: nothing to disclose

M.E. University of Alabama-Birmingham School of Medicine, Birmingham VA Medical Center, Birmingham, AL

Disclosure: nothing to disclose

K.Mautner Department of PM\&R and Department of Orthopedics, Emory Sports Medicine Center, Atlanta, GA

Disclosure: nothing to disclose

K.McInnis Harvard Medical School, Massachusetts General Hospital Sports Medicine Center, Boston, MA

Disclosure: nothing to disclose
J.N. Department of Medicine, Physical Medicine and Rehabilitation, Virginia Tech Carilion School of Medicine, Roanoke, VA Disclosure: nothing to disclose

A.R. Department of Family Medicine, Sports Medicine Section, University of Washington, Seattle, WA

Disclosure: nothing to disclose

B.K. Department of Rehabilitation, University of Washington, and Department of Orthopaedics and Sports Medicine and Pediatrics, Seattle Children's Hospital, Seattle, WA

Disclosure: nothing to disclose 